\title{
Spectroscopic Characterization of Successive Phosphorylation of the Tissue Factor Cytoplasmic Region
}

\author{
Mehmet Sen ${ }^{1,2}$, Mark Herzik Jr. ${ }^{1,3}$, John W. Craft Jr. ${ }^{1}$, Andrea L. Creath ${ }^{1}$, Sameer Agrawal ${ }^{1}$, \\ Wolfram Ruf ${ }^{4}$ and Glen B. Legge ${ }^{*}, 1$ \\ ${ }^{1}$ University of Houston, Department of Biology and Biochemistry, Houston, TX 77204-5001, USA \\ ${ }^{2}$ Present Address: Harvard Medical School, CBR Institute for Biomedical Research, Springer Lab, Boston, MA 02115, \\ USA \\ ${ }^{3}$ Present Address: University of California, Berkeley, Department of Molecular \& Cell Biology, Stanley Hall, Berkeley, \\ CA 94720-3206, USA \\ ${ }^{4}$ The Scripps Research Institute, Department of Immunology, La Jolla, CA 92037, USA
}

\begin{abstract}
Tissue Factor (TF) is well known for its role during the activation of the coagulation pathway, but it is also critical for tumor biology and inflammation through protease activated receptor (PAR) 2 signaling. This signaling function is modulated by the successive phosphorylation of residues Ser253 and Ser258 within the TF cytoplasmic region (TFCR). This paper reports how we used NMR and spectroscopic methods to investigate the structural propensities of the unphosphorylated and phosphorylated forms of the TFCR. When unphosphorylated, the TFCR forms a local hydrophobic collapse around Trp254 and an electropositive patch from the membrane proximal basic block (Arg246-Lys247) to the conserved PKC $\alpha$ consensus residue Lys255. Phosphorylation of Ser253 alters the charge characteristics of this membrane proximal region, thereby strengthening the interaction between residue Ala248 and the Trp254 aromatic group. Phosphorylation of the Ser258-Pro259 motif destabilizes a turn at the C-terminus to form an extended polyproline helical motif. Our data suggests that by changing both its charge and local structural propensity, covalent modifications of the TFCR can potentially regulate its association with the cellular membrane and its signaling partners.
\end{abstract}

\section{INTRODUCTION}

Activation of the coagulation cascade is a major host defense pathway in inflammation and wound repair. Tissue Factor (TF) is the upstream activator of coagulation, functioning as a cell surface receptor for coagulation factor VIIa (VIIa) to allow for recruitment of substrate factor X (Xa) in the ternary coagulation initiation complex [1]. In addition, both the ternary TF-VIIa-Xa and the binary TFVIIa complex have direct signaling functions in angiogenesis and tumor progression [2-4].

TF consists of two extracellular fibronectin type III folds (residues Ser1-Glu219), a single transmembrane helix (residues Ile220-Leu242), and a 21-amino acid cytoplasmic region (TFCR) (residues His243-Ser263) [5-8]. TF is structurally related to type 2 cytokine receptors, but has a cytoplasmic motif alternate to those typically known to mediate tyrosine kinase recruitment. Human TFCR undergoes reversible covalent modifications through either palmitoylation of Cys 245 or phosphorylation of Ser253 and Ser258 [9-12]. The location of the first phosphorylation site is variable, although the latter ( $\mathrm{Ser} / \mathrm{Thr}$ )-Pro phosphoracceptor site is conserved in all mammalian species. This

*Address correspondence to this author at the University of Houston, Department of Biology and Biochemistry, 4800 Calhoun Rd, Houston, TX 77204-5001, USA; Tel: (713) 743 8380; Fax: (713) 743 8351;

E-mail: glegge@uh.edu $\mathrm{p}$ (Ser/Thr)-Pro phosphorylation motif is a potential recognition sequence for the pepidtyl/prolyl isomerase Pin 1 [13]. Phosphorylation by a proline-directed kinase at the conserved Ser258/Pro259 motif is enhanced in vivo when palmitoylation of TFCR is inhibited $[10,14]$.

A functional role for the TFCR was first documented in hematogenous metastasis, and involves phosphorylation of the TFCR [15-18], with phosphorylation observed in accelerated angiogenesis and tumor growth [11,19-21]. Moreover, phosphorylation of the TFCR regulates cell migration specifically mediated by integrin $\alpha_{3} \beta_{1}$ [22], with persistent phosphorylation of TFCR present in cancer and neovascular eye disease [11]. Depalmitoylation and successive phosphorylation of TFCR is essential in mediating the function of TF its interaction with PAR-2 [1012]. Hence, understanding the changes that result from these reversible covalent modifications will highlight their important role in the intrinsic coagulation cascade and TF signaling [23]. In this paper, we use NMR and bioinformatic methods to investigate the structural role of successive TFCR phosphorylation.

\section{MATERIALS AND METHODS}

\section{Tissue Factor Cytoplasmic Region Peptide}

Peptides corresponding to the human TFCR from residues Cys245 to Ser263 (CRKAGVGQS WKENSP LNVS) in its unphosphorylated (TFCR), single phosphorylated at Ser253 (TFSP253) or Ser258 (TFSP258), and double 
phosphorylated (TFPP) forms were synthesized for NMR and functional analysis by The Scripps Research Institute core facility. All samples were purified by reverse phase HPLC, their masses were confirmed by MALDI-TOF mass spectroscopy (2059.3 Da for the TFCR and 2216.3 Da for the TFPP), and were lyophilized.

\section{Extinction Coefficient Calculations}

The extinction coefficients of the four peptides were determined by the Gill and von Hippel method [24]. Absorbance maxima of the peptides were determined at 280 $\mathrm{nm}$ by scanning with a UV-VIS spectrophotometer (Waters, Inc.). The concentration of the peptides was adjusted to approximately $0.1 \mu \mathrm{M}$, which resulted in $\mathrm{Abs}_{280 \mathrm{~nm}}$ of 0.56 , and the denaturant buffer containing $8 \mathrm{M}$ Guanidine Hydrochloride $(\mathrm{Gdn}-\mathrm{HCl})$ and $20 \mathrm{mM}$ phosphate $(\mathrm{pH} 7.2)$ was prepared. $375 \mu \mathrm{L}$ of peptide solution was mixed with $1.125 \mathrm{ml}$ denaturant buffer or $1.125 \mathrm{ml}$ of $20 \mathrm{mM}$ phosphate buffer ( $\mathrm{pH}$ 7.2). $\mathrm{Abs}_{280 \mathrm{~nm}}$ values of peptide samples were recorded in triplicate at room temperature and averaged.

\section{Denaturation Titrations}

A Cary Eclipse Fluorescence Spectrophometer with a slit width of $5 \mathrm{~nm}$ was used for the denaturation studies. The intrinsic fluorescence of Trp254 increased on denaturation, which allowed the unfolding reaction to be monitored. TF peptides were prepared at $5 \mu \mathrm{M}$ in $10 \mathrm{mM}$ phosphate buffer ( $\mathrm{pH}$ 6.0) with degassed urea solution. After the peptide/denaturant solutions were incubated at room temperature for approximately 30 minutes, the maximal change in fluorescence upon denaturation was obtained with an excitation wavelength of $280 \mathrm{~nm}$ and emission wavelength of $350 \mathrm{~nm}$.

\section{Circular Dichroism Spectroscopy}

Circular Dichroism (CD) spectra of the four peptide samples (TFCR, TFSP253, TFSP258 and TFPP) were recorded on an Olis-1000 RSM equipped with a thermoelectric temperature controller. The peptide samples were prepared at $155 \mu \mathrm{M}, \mathrm{pH} 6.0$, and run at $20{ }^{\circ} \mathrm{C}$ in a 1.0 $\mathrm{mm}$ cell. Spectra representing the three average scans from $260 \mathrm{~nm}$ to $187.5 \mathrm{~nm}$ were measured using a step size of 0.87 $\mathrm{nm}$ and a signal-averaging time of 5 seconds.

\section{Homonuclear and Natural Abundance Heteronuclear NMR Data Acquisition}

The IP-COSY [25] experiments were acquired at 600 and $800 \mathrm{MHz}$ Bruker Spectrometers at $285 \mathrm{~K}$ with water suppression achieved by Excitation Sculpting [26]. Homonuclear ${ }^{1} \mathrm{H} /{ }^{1} \mathrm{H}$ TOCSY spectra were acquired with a 70 ms mixing time, whereas both the ${ }^{1} \mathrm{H} /{ }^{1} \mathrm{H}$ NOESY and ROESY spectra were acquired with mixing times of 100 and $300 \mathrm{~ms}$, respectively. The final concentration of each peptide was adjusted to $6 \mathrm{mM}$, as calculated by $\mathrm{Abs}_{280 \mathrm{~nm}}$ in $6 \%(\mathrm{v} / \mathrm{v})$ ${ }^{2} \mathrm{H}_{2} \mathrm{O}$ at $\mathrm{pH}$ 6.0. Spectral widths were $6,830 \mathrm{~Hz}$ with 4,096 complex points in $t_{2}, 6,721 \mathrm{~Hz}$ with 512 complex points in $t_{1}$, 32 transient scans per increment for all spectra and referenced to TSP. Natural abundance heteronuclear 2D ${ }^{1} \mathrm{H} /{ }^{15} \mathrm{~N}$ and ${ }^{1} \mathrm{H} /{ }^{13} \mathrm{C}$ HSQC spectra were acquired with 64 scans for each spectrum. ${ }^{1} \mathrm{H} /{ }^{15} \mathrm{~N}$ HSQC with 2,048 complex points $(11,160 \mathrm{~Hz})$ in $t_{2}$ dimension, and 256 complex points
$(2,594 \mathrm{~Hz})$ in $t_{l}$ and ${ }^{1} \mathrm{H} /{ }^{13} \mathrm{C}$ HSQC with 2,048 complex points $(8,992 \mathrm{~Hz})$ in $t_{2}$ dimension and 256 complex points $(12,703 \mathrm{~Hz})$ in $t_{l}$ dimension were acquired. All NMR spectra were processed and analyzed by NMRPipe [27] and NMRView [28], with zero-filling and a Lorentzian-toGaussian weighing factor applied prior to Fourier transformation.

\section{Peptide Structure Calculations}

Crosspeak volumes in the NOESY and ROESY spectra were binned as distance restraints $(2.7,3.5,5$ and $6 \AA$ ). Initial structures were generated by DYANA [29], and structures were minimized using AMBER8 [30] with chemically correct phosphoserine libraries [31]. The quality of each final structure was analyzed using PROCHECKNMR [32]. The ${ }^{1} \mathrm{H},{ }^{13} \mathrm{C}$ and ${ }^{15} \mathrm{~N}$ resonances of TFCR variants have been deposited in the BioMagResBank www.bmrb.wisc.edu under acces-sion number 6993 for TFPP, 6991 for TFCR, 6996 for TFSP253 and 6998 for TFSP258. The coordinates of the 10 best conformers for each variant are deposited in the Protein Data Bank (PDB) www.rcsb.org under accession codes $2 \mathrm{CEH}$ for TFPP, $2 \mathrm{CEF}$ for TFCR, 2CEZ for TFSP253 and 2CJF for TFSP258.

\section{RESULTS}

\section{Chemical Denaturation Profiles of the TF Peptides by Fluorescence Spectroscopy}

To investigate changes in the local environment of the Trp254 aromatic ring, we monitored the denaturation profiles of each $\mathrm{TF}$ peptides by fluorescence emission spectroscopy at $350 \mathrm{~nm}$ with increasing urea concentrations. The concentration of each peptide was calculated using the native state Extinction Coefficients $\varepsilon_{\text {native }}$ as follows: TFCR $5611 \mathrm{M}^{-1} \mathrm{~cm}^{-1}$; TFSP253 $5789 \mathrm{M}^{-1} \mathrm{~cm}^{-1}$; TFSP258 $5623 \mathrm{M}^{-1}$ $\mathrm{cm}^{-1}$; and TFPP $5848 \mathrm{M}^{-1} \mathrm{~cm}^{-1}$. Denaturation titration profiles (Supplementary Fig. 1) indicate a denaturation midpoint at $\sim 1 \mathrm{M}$ urea for both the TFCR and TFPP peptides, suggesting a common local hydrophobic collapse around Trp254 (Fig. 1A, B).

\section{Spectroscopy}

$\mathrm{CD}$ was used to probe the overall structural propensities in the TF peptides (Fig. 1C), with the TFCR peptide showing a dominant negative peak at $198 \mathrm{nM}$ and a negative shoulder at $222 \mathrm{~nm}$. This minimum was blue shifted to $196 \mathrm{~nm}$ for the TFPP peptide and the shoulder at $215 \mathrm{~nm}$ was abolished. These data indicate the TF peptides are largely disordered or have non-regular structure in the absence of their membrane tether or when not bound to an intracellular ligand. The reduction of the negative shoulder at $222 \mathrm{nM}$ is more evident following phosphorylation of Ser258, rather than phosphorylation of Ser253 (data not shown), which suggests local helical propensity in the C-terminal region of TFCR prior to phosphorylation of the Ser-Pro motif.

\section{NMR Assignment of the TF Peptides}

Each TFCR peptide was assigned by homonuclear NMR [33] (Fig. 2). Complete proton assignments were obtained for each peptide. Both the cis and trans forms of Pro259 were present in the NMR spectra of each TFCR peptide

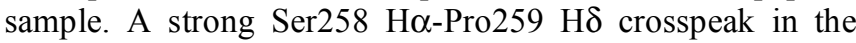




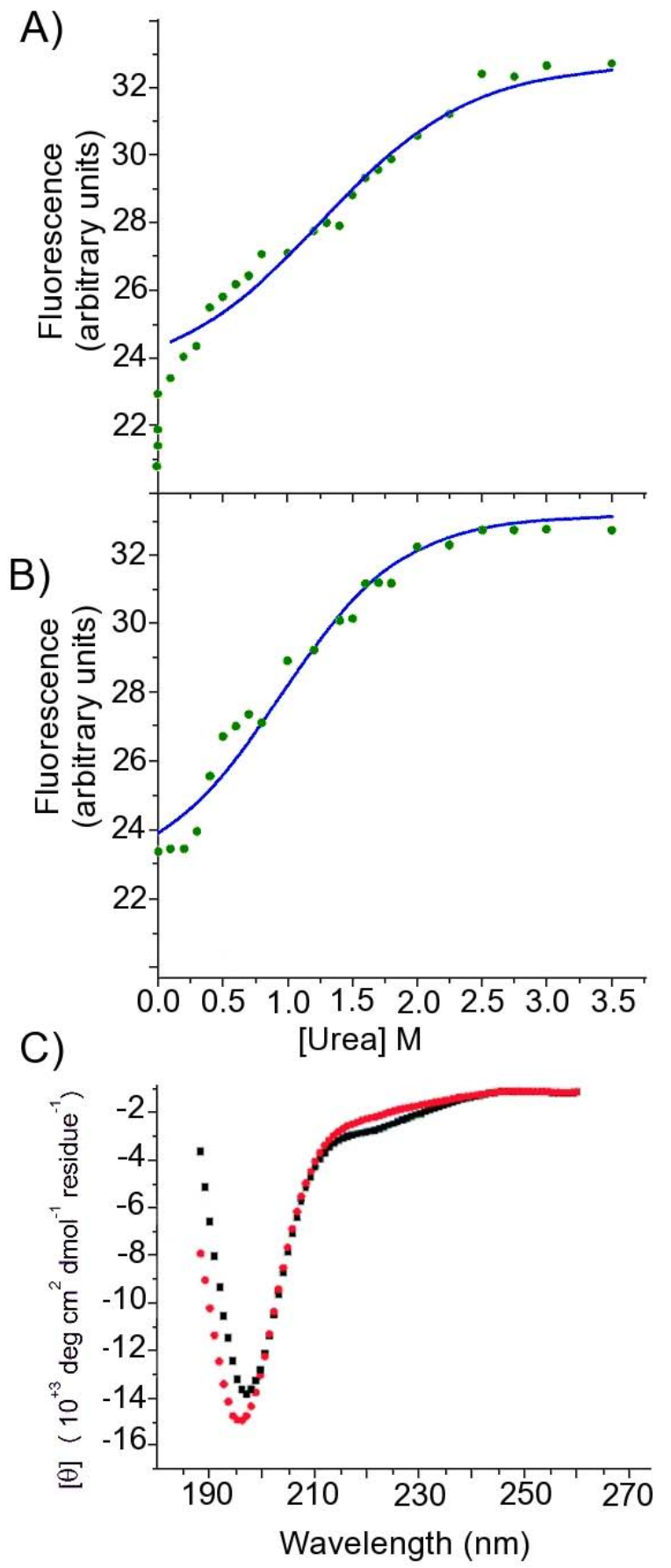

Fig. (1). Intrinsic fluorescence of $5 \mu \mathrm{M}$ TFCR peptides was monitored at $350 \mathrm{~nm}$, with an excitation wavelength of $280 \mathrm{~nm}$ as a function of [Urea] for (A) TFCR (B) TFPP peptides at room temperature in $10 \mathrm{mM}$ sodium phosphate $(\mathrm{pH} 6.0)$. The solid line represents the best fit of the data to a non-linear polynomial regression as calculated by Origin 6.0. (Microcal Software, Inc.). (C) Far UV circular dichroism spectra (188-260 nm) of the $155 \mu \mathrm{M}$ TFCR (black squares) and TFPP (red circles) peptides at $295 \mathrm{~K}$.

ROESY spectra indicated that the dominant form of the Pro259 peptide bond was trans for each of the TF peptides. The phosphorylation-induced changes in chemical shift are illustrated in Fig. (3A), which shows the TFCR-TFPP $\Delta \delta$ (ppm) for both the $\mathrm{H} \alpha$ and $\mathrm{HN}$ atoms. These data indicate that phosphorylation induces significant local changes in the chemical shift for the phosphorylated residues Ser253 and
Ser258, as well as inducing deviations for the more distal residues Ala248 and Ser263.

\section{Heteronuclear Assignments and Local Hydrophobic Collapse of Trp254}

The homonuclear NMR data were used to obtain backbone ${ }^{15} \mathrm{~N}$ and ${ }^{13} \mathrm{C} \alpha$ heteronuclear assignments from the natural abundance ${ }^{1} \mathrm{H} /{ }^{15} \mathrm{~N}$ and ${ }^{1} \mathrm{H} /{ }^{13} \mathrm{C}$ HSQC spectra of the $\mathrm{TF}$ peptides. The ${ }^{13} \mathrm{C} \alpha$ chemical shift deviations from random coil for both the TFCR and TFPP peptides (Fig. 3B) show weakly positive ${ }^{13} \mathrm{C} \alpha \Delta \delta(\mathrm{ppm})$ values for residues between Lys247 and Ser253, as well as Lys255 and Ser258 $\left({ }^{13} \mathrm{C} \alpha \Delta \delta(\mathrm{ppm})>0.5 \mathrm{ppm}\right)$, which suggest a weak helical propensity for these residues. In the ROESY spectra this propensity, which is present in both the phosphorylated and unphosphorylated forms of TFCR, reflects a local hydrophobic collapse of residues Gln252 to Glu256 around the aromatic ring of Trp254. This local collapse is phosphorylation-independent, as crosspeaks between both the Lys255 He methylene and the Glu256 H $\alpha$ and the Trp254 $\mathrm{H} \zeta 2$ and $\mathrm{H \eta} 2$ atoms are present in the ROESY spectra of both the TFCR and TFPP peptides.

To further explore the chemical environment of Trp254, we calculated the NMR solution structures of the TF peptides; a summary of the top 10 structures is presented in Table 1. These structures indicate a local collapse between residues Gln252 to Glu256, with an RMSD of $1.0 \AA$ for all heavy atoms for these residues. This collapse is largely the result of contacts with the aromatic sidechain of Trp254 with the sidechain methylene groups of Lys255 and Glu256, with additional interactions of the $\mathrm{H} \alpha$ and $\mathrm{H} \gamma$ from $\mathrm{Gln} 252$ to pack across one face of the aromatic ring (Figs. 4A, B).

\section{The Effect of Sequential Phosphorylation of TFCR}

Clearly, a change in electrostatics is the dominant role of phosphorylating the Ser253 and Ser258 residues (Figs. 4C, D), and the observed local collapse between residues Gln 252 to Glu256 is phosphorylation independent. However, there are notable differences in the calculated TF peptide structures outside of this conserved region, with these differences at the N-terminal region due to Ser253 phosphorylation and the differences at C-terminal region due to Ser258 phosphorylation. Indeed, our NMR data for the singly phosphorylated peptides indicates that the two phosphorylation sites act independently from each other, but locally behave in a manner similar to that of the double phosphorylated peptide. Namely, phosphorylation of Ser253 neutralizes much of the electropositive charge from Lys255 and allows the Ala248 residue, which neighbors the basic block Arg246 and Lys247 residues, to interact with the Trp254; phosphorylation of Ser258 destabilizes a local $i, i+3$ helical turn that is centered between the Pro259 carbonyl and the Val262 amide.

\section{DISCUSSION}

In this paper, we explore the role of phosphorylation of TFCR by examining the spectral properties of the free peptide that correspond to residues Cys245-Ser263 in the cytoplasmic region of the full-length protein. While recognizing that these studies of the free peptides may not fully account for the tethered nature of the TFCR in vivo or 

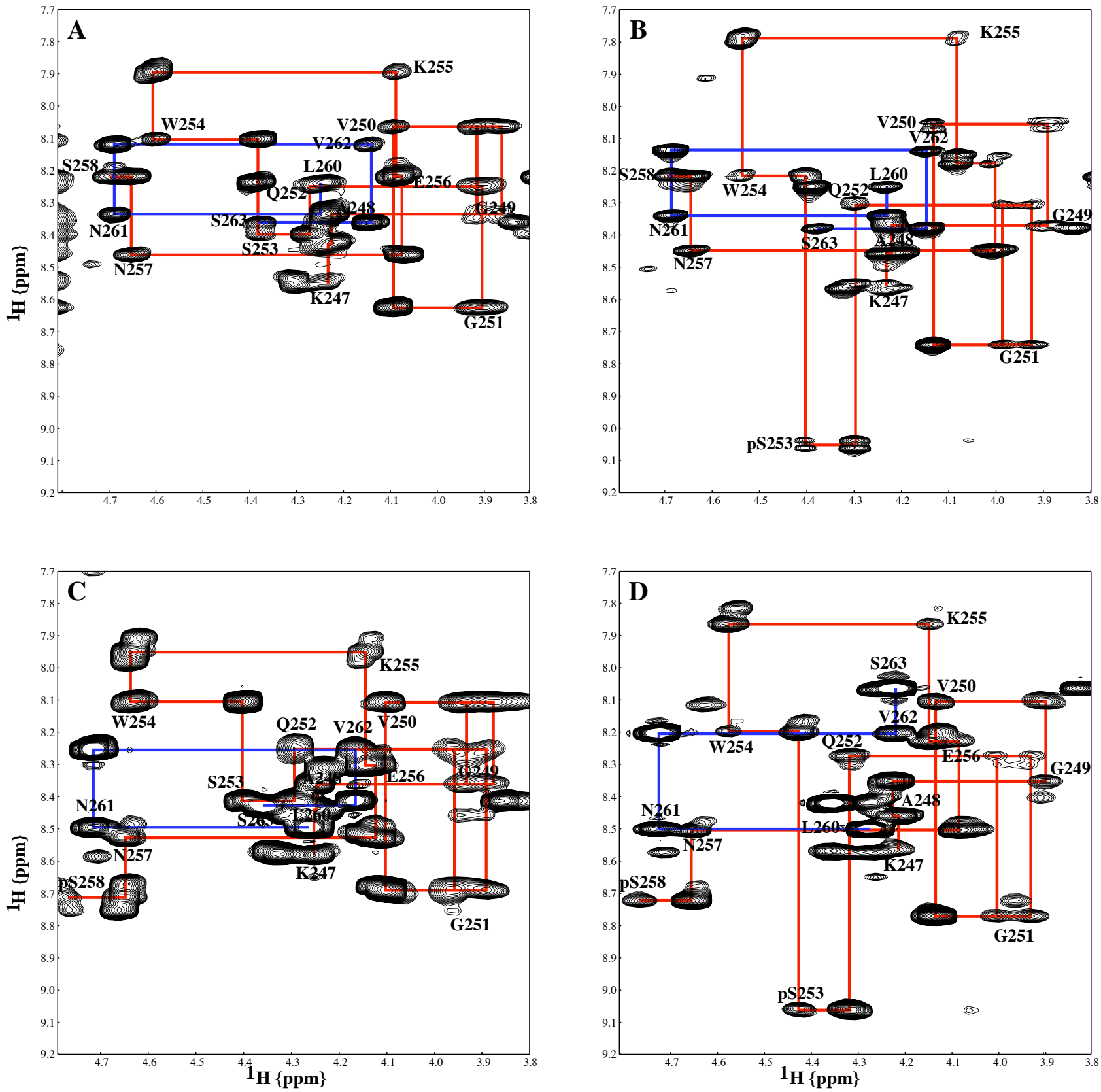

Fig. (2). ROESY ${ }^{1} \mathrm{H} /{ }^{1} \mathrm{H}$ 2D NMR spectra illustrating the sequential backbone assignment of the (A) unphosphorylated, (B) Ser253 phosphorylated, (C) Ser258 phosphorylated, and (D) double phosphorylated TF peptides. HN-H $\alpha$ connectivities prior to Pro259 and after Pro259 are shown with red and blue lines, respectively. Backbone assignments were built by following the $\mathrm{HN}(i)-\mathrm{H} \alpha(i)$ and $\mathrm{HN}(i)-\mathrm{H} \alpha(i-1)$ crosspeaks.

its interaction with the lipid bilayer, we assert that these studies can identify both the local structural propensities and their dependence of the phosphate groups at Ser253 and/or Ser258. These propensities suggest that the unphosphorylated electropositive C-terminal region of the TFCR, together with the palmitoyl group on Cys245, inhibit kinase accessibility and the phosphorylation of Ser258. While we note that in a conventional sense, there is not strong sequence conservation of the TFCR between mammalian species, there are several aspects of this sequence that are conserved; specifically, the highly electropositive basic block with either one or two reversible palmitoylation sites, a fixed ten amino acid separation between the last palmitoylation site and a conserved lysine residue (Lys255 in humans), an overall length for the cytoplasmic region from the last palmitoylation site to the C-terminus of either 18 residues or 19 residues, and at least two phosphorylation sites (Fig. 5).

With the palmitoylation of Cys245 regulating the initial protein kinase $\mathrm{C}(\mathrm{PKC}) \alpha$ dependent phosphorylation of 


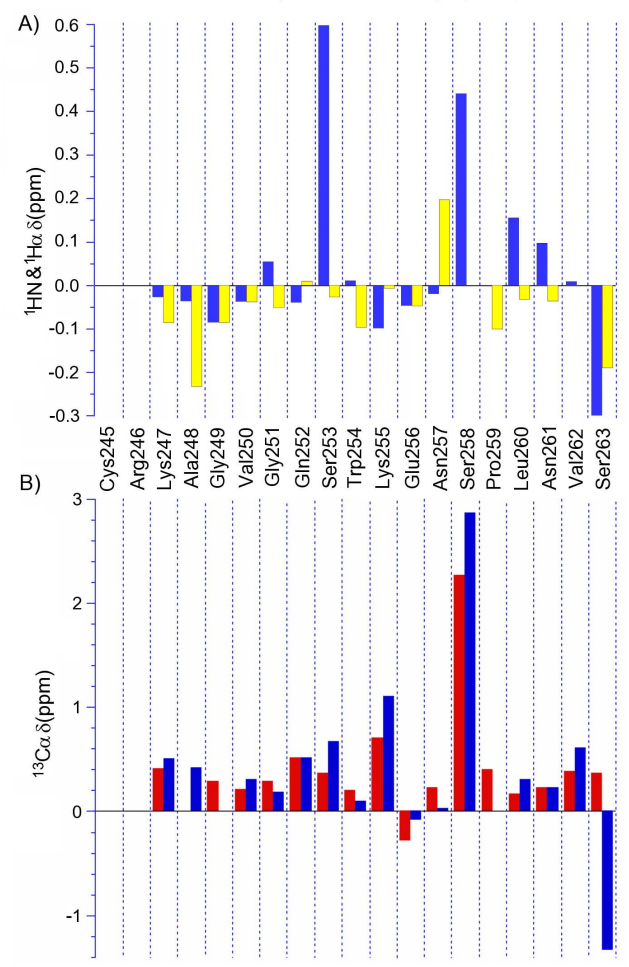

Fig. (3). Chemical shift deviation $\Delta \delta(\mathrm{ppm})$ for TFCR and TFPP peptides. (A) Relative chemical shift deviation $(\Delta \mathrm{ppm})$ of TFCR and TFPP for the ${ }^{1} \mathrm{HN}$ (blue) and ${ }^{1} \mathrm{H} \alpha$ (yellow) the assignments. (B) ${ }^{13} \mathrm{C} \alpha$ deviation from random coil chemical shifts $(\delta \mathrm{ppm})$ for TFCR (red) and TFPP peptides (blue).

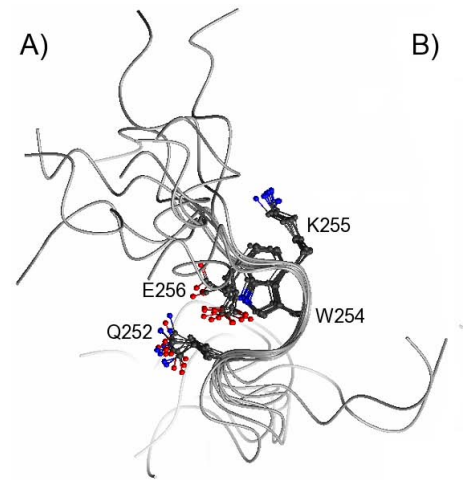

B)
C)

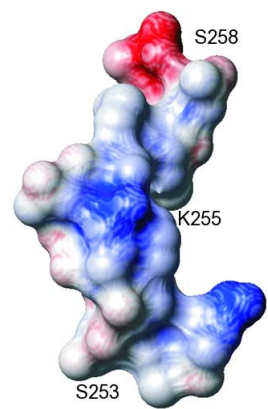

D)

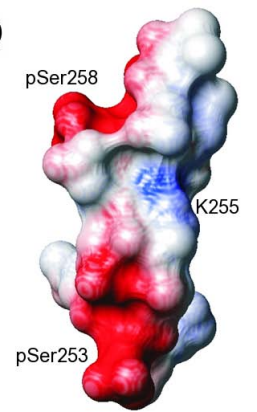

Fig. (4). Superimposed top 10 NMR structures of (A) unphosphorylated and (B) double phosphorylated TFCR, where the side chain heavy atoms of Gln252, Trp254, Lys255 and Glu256 are shown in ball and stick. Electrostatic surface plots of (C) unphosphorylated (D) double phosphorylated TFCR peptides lowest energy structures. Figures were generated by MOLMOL [41].

Table 1. NMR and Refinement Statistics for Top Ten Minimized DYANA Structures Calculated for the Unphosphorylated (TFCR), Singly Phosphorylated (TFSP253 and TFSP258) and Double Phosphorylated (TFPP) Peptides

\begin{tabular}{|c|c|c|c|c|}
\hline & TFCR & TFPP & TFSP253 & TFSP258 \\
\hline \multicolumn{5}{|l|}{ NMR distance \& dihedral constraints } \\
\hline Total NOE & 55 & 49 & 58 & 55 \\
\hline Sequential $(|\mathrm{i}-\mathrm{j}|=1)$ & 27 & 26 & 26 & 32 \\
\hline Medium-range $(|\mathrm{i}-\mathrm{j}| \leq 4)$ & 27 & 19 & 28 & 19 \\
\hline Long-range $(|\mathrm{i}-\mathrm{j}| \geq 5)$ & 1 & 4 & 4 & 4 \\
\hline$\chi 1$ angle restraints & 3 & 3 & 3 & 3 \\
\hline \multicolumn{5}{|l|}{ Structure Statistics (10 structures) } \\
\hline Mean distance constraints $(\AA)$ & 0.34 & 0.26 & 0.36 & 0.21 \\
\hline Mean dihedral angle constraints $\left({ }^{\circ}\right)$ & 5.8 & 1.9 & 6.2 & 32.0 \\
\hline Max. dihedral angle violation $\left({ }^{\circ}\right)$ & 5.8 & 1.9 & 6.2 & 32.0 \\
\hline Max. distance constraint violation $(\AA)$ & 0.34 & 0.48 & 0.38 & 0.24 \\
\hline \multicolumn{5}{|l|}{ Energies } \\
\hline Mean Amber Energy (kcal/mol) & -560.1 & -919.4 & -734.0 & -729.3 \\
\hline Mean Constraints Violation Energy (kcal/mol) & 3.2 & 5.4 & 9.5 & 6.8 \\
\hline \multicolumn{5}{|l|}{ Pairwise R.M.S.D. ( $(\AA)$ Res. (Ala248-Pro259) } \\
\hline Heavy & 2.5 & 1.0 & 1.5 & 1.2 \\
\hline Backbone & 2.2 & 0.6 & 1.1 & 1.2 \\
\hline \multicolumn{5}{|l|}{ Procheck statistics for $\varphi$ and $\psi$} \\
\hline Residues in allowed region & 88.6 & 90.0 & 96.4 & 88.6 \\
\hline Residues in disallowed region & 11.4 & 10.0 & 3.6 & 11.4 \\
\hline
\end{tabular}


Ser253, and then subsequent phosphorylation of the Ser258Pro259 motif by a proline-directed kinase [10], a model of the successive role of TFCR covalent modifications and PAR2 signaling is emerging [12]. Lys255 is essential in mediating the phosphorylation of TFCR [34], which follows depalmitoylation of Cys245 [10]. Hence, our primary sequence alignment (Fig. 5), which shows a conserved ten residue distance between the palmitoylation sites and the conserved lysine is notable. Unlike previously published alignments [23,34], our fixed ten-residue distance between the intracellular lipid anchor (palmitoylated Cys245) and the PKC $\alpha$ consensus residue (Lys255) is consistent with a model of reversible palmitoylation that modulates substrate recognition and accessibility of the PKC $\alpha$ kinase and initial phosphorylation of Ser253 [10,35].

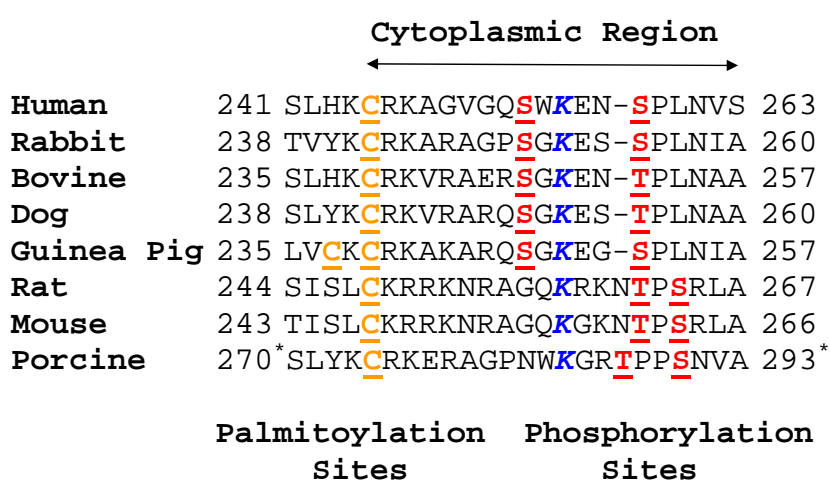

Fig. (5). Primary sequence alignment of Human, Rabbit, Guinea Pig, Bovine, Dog, Pig, Rat and Mouse TFCR. Points of covalent modification are underlined, while the cysteine palmitoylation sites are highlighted orange and the two phosphorylation sites are highlighted red and the PKC $\alpha$ consensus sequence lysine is shown in blue text. The black arrow indicates the location of the human Cys245Ser263 TF peptides used in these peptide studies. Residue numbering for all sequences, except Pig, is based on the processed polypeptide and does not include the signal sequence.

For all TFCR sequences, the positively-charged basic block residues would interact favorably with the negatively charged intracellular membrane [36] and would thus promote a favorable extended interaction between the two $[37,38]$. Hence, both palmitoylation of Cys245 and extended electrostatic interactions may act in concert to prevent $\mathrm{PKC} \alpha$ accessibility to the Ser253. Conversely, although depalmitoylation of Cys245 would not affect the overall positive charge of this $\mathrm{N}$-terminal region of TFCR, the absence of this hydrophobic anchor would weaken any extended interactions of the TFCR with the membrane and expose the conserved $i+10 \mathrm{PKC} \alpha$ consensus sequence residue, Lys255, for recruitment of the kinase and phosphorylation of Ser253.

The NMR data for the Ser253 phosphorylated peptides indicate their $\mathrm{N}$-terminal regions were more compact than the unphosphorylated peptides (Table 1). This may be due to charge neutralization of this region (Fig. 4C), which stabilizes the interaction of the Ala248 methyl group and the Arg247-Ala248 and Ala248-Gly249 peptide bonds with the Trp254 aromatic ring. The phosphorylation of Ser253 would further destabilize any extended association of the TFCR with the electronegative phospholipid bilayer. Therefore, both depalmitoylation of Cys245 and phosphorylation of
Ser253 may act in concert to reduce the interaction of the TFCR with the bilayer to promote the recruitment of a proline directed kinase and phosphorylation of Ser258.

The NMR and CD spectroscopic data indicate that a short helical turn is formed by residues Pro259-Val262 at the Cterminus of the unphosphorylated TFCR. The primary sequence alignment (Fig. 5) favors the presence of alanine residues towards the $\mathrm{C}$-terminus, which reveal the structural preference of a phosphate inhibited ligand. This local turn is disrupted by the phosphorylation of the serine/threonineproline motif to form an extended polyproline II helix conformation, which is a structural motif recognized by the Pin1 WW domain [39]. Within this C-terminal region, the Ser/Thr-Proline motif is strongly conserved and is often neighboring leucine residues. Upon phosphorylation of the conserved Ser-Pro motif, an extended polyproline II helix is formed by three contiguous residues that favor this conformation (pSer258-Pro259-Leu260 in humans) [40]. These local phosphate-dependent structural propensities within the TFCR suggest a role in regulating substrate recruitment and TF signaling. Our work will enhance future studies to delineate how persistent TFCR phosphorylation is correlated to disease states, such as accelerated angiogenesis and tumor growth.

\section{ACKNOWLEDGEMENTS}

This work was supported by grants from the National Institutes of Health (PO1 HL016411) and the Welch Foundation (E-1580). We thank Viet and Quoc Phuong for their technical work.

\section{REFERENCES}

[1] Norledge BV, Petrovan RJ, Ruf W, Olson AJ. The tissue factor/factor VIIa/factor Xa complex: a model built by docking and site-directed mutagenesis. Proteins 2003; 53(3): 640-8.

[2] Riewald M, Ruf W. Mechanistic coupling of protease signaling and initiation of coagulation by tissue factor. Proc Natl Acad Sci USA 2001; 98(14): 7742-47.

[3] Ahamed J, Belting M, Ruf W. Regulation of tissue factor-induced signaling by endogenous and recombinant tissue factor pathway inhibitor 1. Blood 2005; 105(6): 2384-91.

[4] Rao LV, Pendurthi UR. Tissue factor-factor VIIa signaling. Arterioscler Thromb Vasc Biol 2005; 25(1): 47-56.

[5] Ruf W, Edgington TS. Structural biology of tissue factor, the initiator of thrombogenesis in vivo. FASEB J 1994; 8(6): 385-90.

[6] Harlos K, Martin DM, O'Brien DP, et al. Crystal structure of the extracellular region of human tissue factor. Nature 1994; 370(6491): 662-6.

[7] Banner DW, D'Arcy A, Chene C, et al. The crystal structure of the complex of blood coagulation factor VIIa with soluble tissue factor. Nature 1996; 380(6569): 41-6.

[8] Donate F, Kelly CR, Ruf W, Edgington TS. Dimerization of tissue factor supports solution-phase autoactivation of factor VII without influencing proteolytic activation of factor X. Biochemistry 2000; 39(37): 11467-76.

[9] Ott I, Fischer EG, Miyagi Y, Mueller BM, Ruf W. A role for tissue factor in cell adhesion and migration mediated by interaction with actin-binding protein 280. J Cell Biol 1998; 140(5): 1241-53.

[10] Dorfleutner A, Ruf W. Regulation of tissue factor cytoplasmic domain phosphorylation by palmitoylation. Blood 2003; 102(12): 3998-4005.

[11] Belting M, Dorrell MI, Sandgren S, et al. Regulation of angiogenesis by tissue factor cytoplasmic domain signaling. Nat Med 2004; 10(5): 502-9.

[12] Ahamed J, Ruf W. Protease-activated receptor 2-dependent phosphorylation of the tissue factor cytoplasmic domain. J Biol Chem 2004; 279(22): 23038-44. 
[13] Landrieu I, Odaert B, Wieruszeski JM, et al. p13(SUC1) and the WW domain of PIN1 bind to the same phosphothreonine-proline epitope. J Biol Chem 2001; 276(2): 1434-38.

[14] Zioncheck TF, Roy S, Vehar GA. The cytoplasmic domain of tissue factor is phosphorylated by a protein kinase C-dependent mechanism. J Biol Chem 1992; 267(6): 3561-64.

[15] Ruf W, Mueller BM. Thrombin generation and the pathogenesis of cancer. Semin Thromb Hemost 2006; 32(Suppl 1): 61-8.

[16] Mueller BM, Ruf W. Requirement for binding of catalytically active factor VIIa in tissue factor-dependent experimental metastasis. J Clin Invest 1998; 101(7): 1372-78.

[17] Bromberg ME, Konigsberg WH, Madison JF, Pawashe A, Garen A. Tissue factor promotes melanoma metastasis by a pathway independent of blood coagulation. Proc Natl Acad Sci USA 1995; 92(18): 8205-9.

[18] Bromberg ME, Sundaram R, Homer RJ, Garen A, Konigsberg WH. Role of tissue factor in metastasis: functions of the cytoplasmic and extracellular domains of the molecule. Thromb Haemost 1999; 82(1): 88-92.

[19] Versteeg HH, Schaffner F, Kerver M, et al. Inhibition of tissue factor signaling suppresses tumor growth. Blood 2007; 111(1): 190-9.

[20] Abe K, Shoji M, Chen J, et al. Regulation of vascular endothelial growth factor production and angiogenesis by the cytoplasmic tail of tissue factor. Proc Natl Acad Sci USA 1999; 96(15): 8663-8

[21] Hembrough TA, Swartz GM, Papathanassiu A, et al. Tissue factor/factor VIIa inhibitors block angiogenesis and tumor growth through a nonhemostatic mechanism. Cancer Res 2003; 63(11): 2997-3000.

[22] Dorfleutner A, Hintermann E, Tarui T, Takada Y, Ruf W. Crosstalk of integrin alpha3beta 1 and tissue factor in cell migration. Mol Biol Cell 2004; 15(10): 4416-25.

[23] Egorina EM, Sovershaev MA, Osterud B. Regulation of tissue factor procoagulant activity by post-translational modifications. Thromb Res 2008; 122(6): 831-7.

[24] Gill SC, von Hippel PH. Calculation of protein extinction coefficients from amino acid sequence data. Anal Biochem 1989; 182(2): 319-26.

[25] Xia Y, Legge G, Jun KY, Qi Y, Lee H, Gao X. IP-COSY. A totally in-phase and sensitive COSY experiment. Magn Reson Chem 2005; 43(5): 372-79.

[26] Hwang TL, Shaka AJ. Multiple-pulse mixing sequences that selectively enhance chemical exchange or cross-relaxation peaks in high-resolution NMR spectra. J Magn Reson 1998; 135(2): 280-7.
[27] Delaglio F, Grzesiek S, Vuister GW, Zhu G, Pfeifer J, Bax A. NMRPipe: a multidimensional spectral processing system based on UNIX pipes. J Biomol NMR 1995; 6(3): 277-93.

[28] Johnson BA. Using NMRView to visualize and analyze the NMR spectra of macromolecules. Methods Mol Biol 2004; 278: 313-52.

[29] Guntert P, Mumenthaler C, Wüthrich K. Torsion angle dynamics for NMR structure calculation with the new program DYANA. J Mol Biol 1997; 273(1): 283-98.

[30] Ponder JW, Case DA. Force fields for protein simulations. Adv Protein Chem 2003; 66: 27-85.

[31] Craft JW, Jr, Legge GB. An AMBER/DYANA/MOLMOL phosphorylated amino acid library set and incorporation into NMR structure calculations. J Biomol NMR 2005; 33(1): 15-24.

[32] Laskowski RA, Rullmannn JA, MacArthur MW, Kaptein R, Thornton JM. AQUA and PROCHECK-NMR: programs for checking the quality of protein structures solved by NMR. J Biomol NMR 1996; 8(4): 477-86.

[33] Wüthrich K. NMR of Proteins and Nucleic Acids. New York: Wiley 1986.

[34] Zioncheck TF, Roy S, Vehar GA. The cytoplasmic domain of tissue factor is phosphorylated by a protein kinase C-dependent mechanism. J Biol Chem 1992; 267(6): 3561-64.

[35] Bach RR. Tissue factor encryption. Arterioscler Thromb Vasc Biol 2006; 26(3): 456-61.

[36] Bach R, Konigsberg WH, Nemerson Y. Human tissue factor contains thioester-linked palmitate and stearate on the cytoplasmic half-cystine. Biochemistry 1988; 27(12): 4227-31.

[37] Sansom MS, Bond PJ, Deol SS, Grottesi A, Haider S, Sands ZA. Molecular simulations and lipid-protein interactions: potassium channels and other membrane proteins. Biochem Soc Trans 2005; 33(Pt 5): 916-20.

[38] Yau WM, Wimley WC, Gawrisch K, White SH. The preference of tryptophan for membrane interfaces. Biochemistry 1998; 37(42): 14713-8.

[39] Zarrinpar A, Lim WA. Converging on proline: the mechanism of WW domain peptide recognition. Nat Struct Biol 2000; 7(8): 61113 .

[40] Bielska AA, Zondlo NJ. Hyperphosphorylation of tau induces local polyproline II helix. Biochemistry 2006; 45(17): 5527-37.

[41] Koradi R, Billeter M, Wüthrich K. MOLMOL: a program for display and analysis of macromolecular structures. J Mol Graph 1996; 14(1): 51-32. 